\title{
Current State of Combined EDS-WDS Quantitative X-Ray Mapping
}

\author{
Ken Moran ${ }^{1}$ and Richard Wuhrer ${ }^{2}$ \\ ${ }^{1}$ Moran Scientific Pty Ltd, 4850 Oallen Ford Road, Bungonia, NSW, 2580, Australia. \\ ${ }^{2}$ Western Sydney University, Advanced Materials Characterisation Facility (AMCF), Australia.
}

Two major problems for quantitative x-ray mapping (QXRM) are interpretation of results under nonideal conditions (strong overlap and small peak size relative to background), and the time required to obtain a good quality x-ray map. Quantitative means that the elements are fully background corrected, overlap corrected and inter-element corrected. With the development of single and multiple Silicon Drift Detectors (SDDs), x-ray mapping at count rates of $100 \mathrm{Kcps}$ to $1,000 \mathrm{Kcps}$ output are possible at optimal energy resolution. With a single Energy Dispersive Spectrometer (EDS) detector at 200kcps output, a 512x512 quantitative map can be obtained in around 4 to 7 hours for major elements $>1 \mathrm{wt} \%$ evenly distributed and minor elements $>0.1 \mathrm{wt} \%$ localised. Thus a $512 \times 512$ x-ray map performed in 30 minutes, gives major elements $>10 \mathrm{wt} \%$ evenly distributed and minor elements $>1 \mathrm{wt} \%$ localised. The ability to have very large collection efficiencies resulting in much higher count rates and their ability to maintain resolution performance means that their sensitivity is vastly superior to $\mathrm{Si}(\mathrm{Li})$ detectors. This has led to the subsequent abandonment of the standard $\mathrm{Si}(\mathrm{Li})$ detector from the new equipment list, even with the poorer performance for $\mathrm{x}$-rays $>10 \mathrm{keV}$ compared to a $\mathrm{Si}(\mathrm{Li})$ detector. Although we have not quite reached "live" x-ray images we are now able to view moderate resolution x-ray images in very short times. SDD's have improved our EDS mapping to such an extent that we can now map $4 \mathrm{k} x$ 4k. If the $\mathrm{x}$-ray volume is say 2.5 micron, then this allows an area of $10 \mathrm{~mm} \times 10 \mathrm{~mm}$ to be mapped in a single pass.

The performance of SDD's has almost removed the need for multiple detectors. Except that two detectors 180 degrees apart, or three detectors 120 degrees apart, are important for indicating problems with rough samples and/or x-ray volume sensitive boundary regions. For QXRM to work correctly for multi-detector systems, the characteristics of each detector must be accurately determined so that the final quantification of the individual detectors can be summed. To accomplish this more effectively, the full spectrum at each pixel for each detector needs to be saved [1]. As a final check for consistency between detectors, a technique was developed called "Colouring Verification Technique" that involves assigning a different Red, Green and Blue (RGB) colour for each detector for the same element [2]. When combining the three maps of the same element, a grey scale map should be obtained not a colour map. This indicates a total correlation between the three detectors at the most critical final stage of quantification. Thus, the use of multiple detectors is an easy way to determine which errors may be inherent in the specimen, the mathematical processing and the detector systems. The use of this colouring technique for multiple detectors has also been found to be very valuable when analysing rough samples.

We are currently improving Wavelength Dispersive Spectrometer (WDS) mapping through the replacement of Proportional Counters (PCs) with SDDs Figure 1. If we incorporate an SDD in the light element range we can improve our detection limit for these elements, and in addition, get improvements in the mid range elements. Figure 2. showing the EDS spectrum for $0.7 \mathrm{wt} \%$ Carbon compared with $8.5 \mathrm{wt} \%$ Carbon. With the SDD, it is possible to get more reliable results with a lot less drift in gain (output voltage versus count rate) that we normally get with PC's.

A good WDS operator must be very meticulous, monitoring items like gas purity, gas flow control, weather pressure patterns, noise levels of baseline and window voltage levels, count rate suppression, anode wire contamination, pinched gas leads, etc. The list goes on. Our current work demonstrates 
the dramatic superiority of SDD over PC (Whether Gas Flow or Sealed). With a proportional counter you have no real idea of what is being counted by the detector. An SDD immediately brings to light problems such as secondary fluorescence, electron interference and other artefacts Figure 3. Often with a PC the extra noise is similar on the peak as the background and so is removed by background subtraction, but the P/B ratio can be significantly reduced. Most PCs have an order of magnitude poorer energy resolution than the SDD, this means that if interferences are present you can get a corresponding improvement in background by removing them either by energy discrimination or physically removing their source. It is important to know whether they are there, so that you can remove them to improve your performance. Imagine the simplicity of a WDS without the need for PC's. It currently costs nearly the same for SDD hardware compared to PC hardware.

References:

[1] K. Moran and R. Wuhrer, "Quantitative Bulk and Trace Element X-Ray Mapping Using Multiple Detectors", Mikrochimica Acta, Vol. 155, pp. 59-66 (2006).

[2] R. Wuhrer and K. Moran, "Dedicated X-Ray Mapping System with Single and Multiple SDD Detectors for Quantitative X-Ray Mapping and Data Processing-ray Mapping and Chemical Phase Mapping with an Amptek SDD”, Microsc. Microanal. 21 (Suppl 3), 2015, p2191-2192.
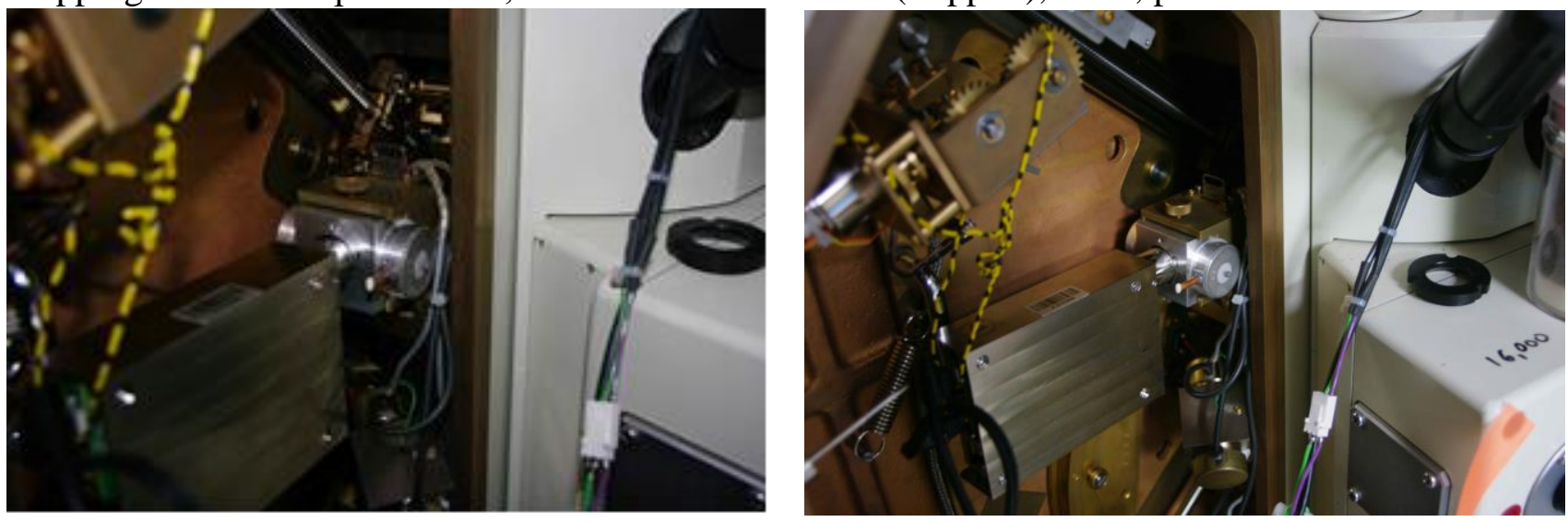

Figure 1. Photographs of Amptek 123 EDS mounted in a JEOL spectrometer through an old proportional counter fitting (PC), which has been bored out to allow alignment of the EDS.

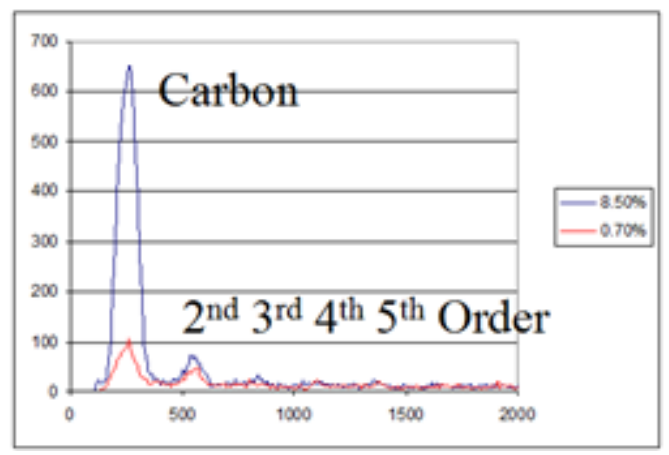

Figure 2. Red and Blue spectra represent $0.7 \mathrm{wt} \%$ and $8.5 \mathrm{wt} \%$ Carbon as measured using an STE crystal $15 \mathrm{keV}$ 50nA.. The spectra also include high order diffraction, secondary fluorescence, high-energy electrons and continuum.

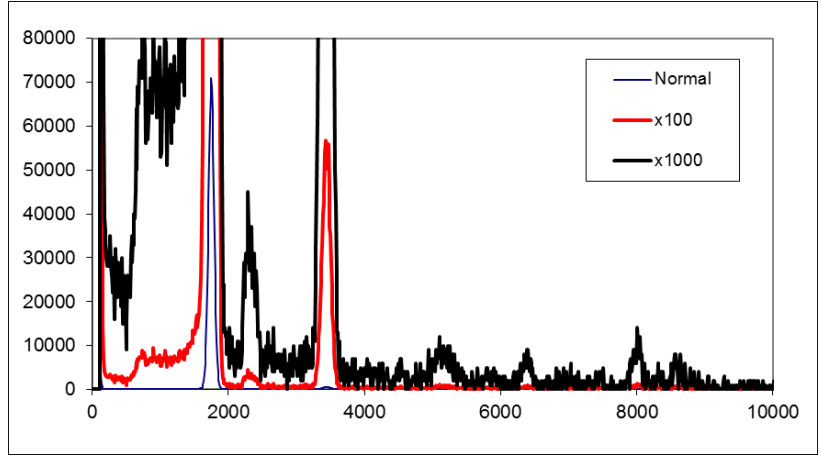

Figure 3. 100\% Si spectrum with 3 different vertical expansions, showing sum peaks (plus high order diffraction), secondary fluorescence $(\mathrm{Pb}, \mathrm{Fe}, \mathrm{Cu}$ and $\mathrm{Zn})$, high-energy electrons and continuum. Measured at $20 \mathrm{keV}, 45 \mathrm{nA}$ TLAP crystal. $18,500 \mathrm{cps}$ on peak (20k total). The $\mathrm{x} 2$ sum peak is $1.2 \%$ of the primary peak. 